\title{
Management of Tooth Wear: An Interdisciplinary Approach
}

\author{
${ }^{1}$ Tsz M Leung, ${ }^{2}$ Michael G Botelho, ${ }^{3}$ Yanqi Yang, ${ }^{4}$ Edward HT Ho
}

\begin{abstract}
Aims: This case report aims to describe a conservative interdisciplinary approach to restore generalized tooth wear complicated with malalignment of teeth.
\end{abstract}

Introduction: Tooth wear is the surface loss of dental hard tissue, i.e., usually multifactorial in origin, excluding other than caries, trauma, or developmental disorder. Severe tooth wear impact the appearance, comfort, function, or longevity of the tooth. Prosthodontic management of patients with tooth wear has been well documented, although prosthodontic treatment is designed to correct minor malocclusion, patients with moderate-to-severe malocclusion accompanied by generalized tooth wear may require preprosthetic orthodontic intervention.

Case report: This case report describes a 47-year-old male patient with generalized tooth wear complicated with lack of interocclusal space and malalignment of teeth. Preventive measures were carried out to address the etiological factors of tooth wear. After orthodontic treatment, definitive restoration was placed in reorganized occlusion at an increased vertical dimension, which restored esthetic and function.

Conclusion: In this clinical report, generalized tooth wear with insufficient space for restoration, complicated by malalignment of teeth, was managed by way of an interdisciplinary approach which allowed a conservative full-mouth rehabilitation. After orthodontic treatment, a reorganized restorative approach was undertaken at an increased vertical dimension, providing an esthetic and functional solution. An occlusal night guard was provided to protect the dental restorations.

Clinical significance: Interdisciplinary approach allows a conservative management for patient with tooth wear complicated by malalignment of teeth.

Keywords: Case reports, Interdisciplinary, Prosthodontic management, Tooth wear.

How to cite this article: Leung TM, Botelho MG, Yang Y, Ho EHT. Management of Tooth Wear: An Interdisciplinary Approach. Int J Experiment Dent Sci 2016;5(2):139-145.

\footnotetext{
${ }^{1}$ Postgraduate Student, ${ }^{2}$ Clinical Associate Professor, ${ }^{3}$ Clinical Assistant Professor, ${ }^{4}$ Part-time Clinical Lecturer

${ }^{1,2,4}$ Department of Oral Rehabilitation, Faculty of Dentistry The University of Hong Kong, Hong Kong, People's Republic of China

${ }^{3}$ Department of Orthodontics, Faculty of Dentistry, The University of Hong Kong, Hong Kong, People's Republic of China

Corresponding Author: Michael G Botelho, Clinical Associate Professor, Department of Oral Rehabilitation, Faculty of Dentistry The University of Hong Kong, Hong Kong, People's Republic of China, Phone: +85228590412, e-mail: botelho@hku.hk
}

\section{Source of support: Nil}

\section{Conflict of interest: None}

\section{INTRODUCTION}

Tooth wear is a normal physiological process during aging, whereas pathological wear happens when the normal wear rate is accelerated by endogenous or exogenous factors. ${ }^{1}$ Traditionally, tooth wear is classified as attrition, abrasion, erosion, and abfraction. Among these, erosion has been identified as the most significant cause of tooth wear and implicated in $89 \%$ of tooth wear cases. ${ }^{2}$ The most current dental literature defines erosion as the loss of tooth substance caused by action of acid unrelated to bacterial action. However, erosion itself is not a chemical mechanism; it is a physical mechanism causing wear by friction from the movement of fluid. Moreover, the term "erosion" has failed to account for the proteolysis and piezoelectric effect, which are also involved in the degradation of tooth substance. Therefore, recently, the term "corrosion" or "biocorrosion" has been proposed. Biocorrosion is defined as the chemical, biochemical, or electrochemical action that causes the degradation of a living tissue. ${ }^{3}$ Apart from acid, biochemical proteolytic enzymes, such as protease from plaque microorganisms and gingival crevicular fluid and pepsin from the stomach and trypsin from the pancreas, can also cause degradation of the dentin organic matrix. ${ }^{4-6}$ In addition, the ability of converting mechanical stress into electrical signal or piezoelectric effect has been observed in dentin, and the electrochemical action of piezoelectric effect on dentin has been shown to cause removal of calcium ions. ${ }^{78}$

Diet, parafunctional habit, and diseases, such as eating disorders or gastric reflux are also important contributing factors of tooth wear. As the causes of tooth wear are multifactorial, ${ }^{9}$ it is difficult to isolate a single etiological factor. Nevertheless, comprehensive history-taking, including dental and medical history, family and social history, thorough intra- and extra-oral examination, and dietary analysis, is crucial to identify and eliminate the possible causes.

After identification of possible etiological factors, an appropriate treatment plan can be established. For many cases of tooth wear, preventive management and monitoring may suffice, ${ }^{10}$ however, more harmful effects will impact the appearance, comfort, function, 
or longevity of the tooth such that active restorative intervention will be indicated.

One of the major challenges in managing advanced tooth wear is the lack of interocclusal space for final restorations. In situation where tooth wear may be localized to certain regions or teeth in the mouth, a more customized restorative approach can be undertaken. The use of a removable cobalt-chromium anterior platform to generate interocclusal space was originally described by Dahl et al. ${ }^{11}$ While an overall increase in the occlusal vertical dimension (OVD) can create space for dental restorations, ${ }^{12}$ it has been shown that patients adapt to moderate changes in OVD provided that occlusal harmony was maintained. ${ }^{13}$ However, this commits the patient to a whole-mouth rehabilitation, which has significant biological and financial cost.

Malocclusion complicates management of tooth wear. To achieve ideal tooth form and function, more tooth structure has to be removed to compensate for the malalignment of teeth. Preprosthetic orthodontic treatment provides a more conservative treatment. Aligning the teeth first can reduce the amount of tooth preparation and it can also improve oral hygiene and provide a healthier periodontium. ${ }^{14}$

The current clinical case reports an interdisciplinary approach for tooth wear management. The patient first underwent a preventive phase and then he received orthodontic appliance to align the teeth. The esthetic outcome was then evaluated using removable acrylic overlay denture and temporary resin restorations. Eventually, the teeth were restored using ceramic and metal restorations.

\section{CASE REPORT}

A 47-year-old Chinese male was referred to the Department of Oral Rehabilitation, Faculty of Dentistry, The University of Hong Kong, for tooth wear management. The patient's chief complaints were tooth hypersensitivity, short teeth, and replacement of his missing teeth. The medical history was unremarkable. He smoked approximately 10 cigarettes per day and nocturnal bruxism was reported.

Extraorally, the patient's face was symmetrical with no temporomandibular abnormality. Intraoral examination showed a permanent dentition with missing 35, 36, and 46. There were signs of generalized tooth wear and dentin exposure, the wear was greater on the palatal surfaces of anterior teeth and occlusal surfaces of posterior teeth, with cupping occlusally on the posterior teeth. A full gold crown was present on the maxillary right second molar, 11 and 21 were rotated, the upper and lower incisors were retroclined, and crowding was present on both the upper and lower anterior segments (Figs $1 \mathrm{~A}$ to C).
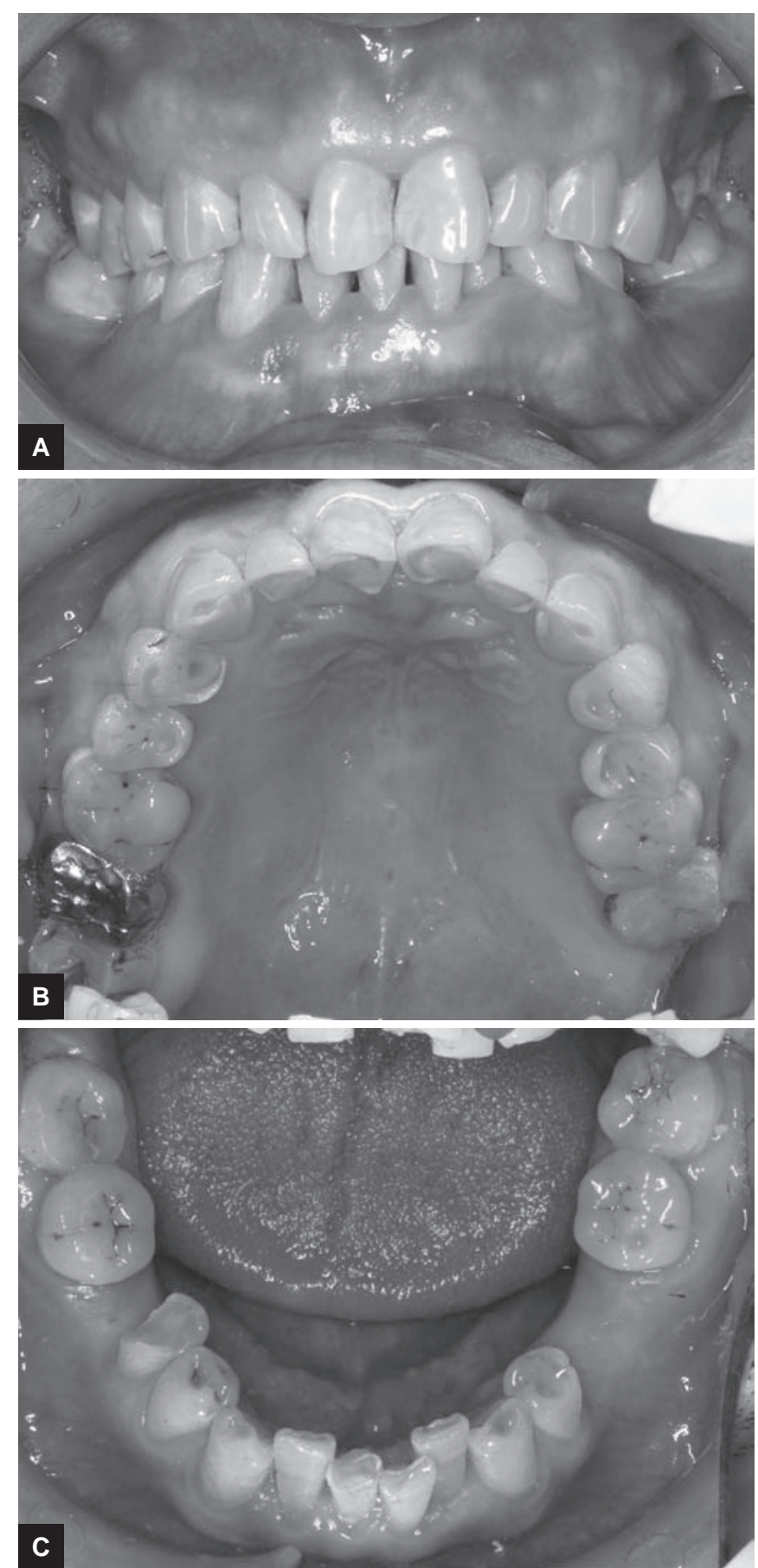

Figs $1 A$ to $C$ : (A) Preoperative frontal view shows worn of incisal edge of maxillary anterior teeth, (B) preoperative upper occlusal view shows mild-to-moderate tooth wear with dentin exposed, and (C) preoperative lower occlusal view shows crowding of lower anterior teeth and mild-to-moderate tooth wear with dentin exposed

All teeth except 17 were responsive to vitality testing. Dietary analysis revealed daily consumption of two to three bottles of sports drinks. The diagnosis of the patient revealed they were partially dentate, malaligned teeth, and with generalized tooth surface loss. The pattern of wear together with diet habit indicated a corrosive component in the diet that contributed to the loss of tooth structure, and with bruxism as a contributing factor. 


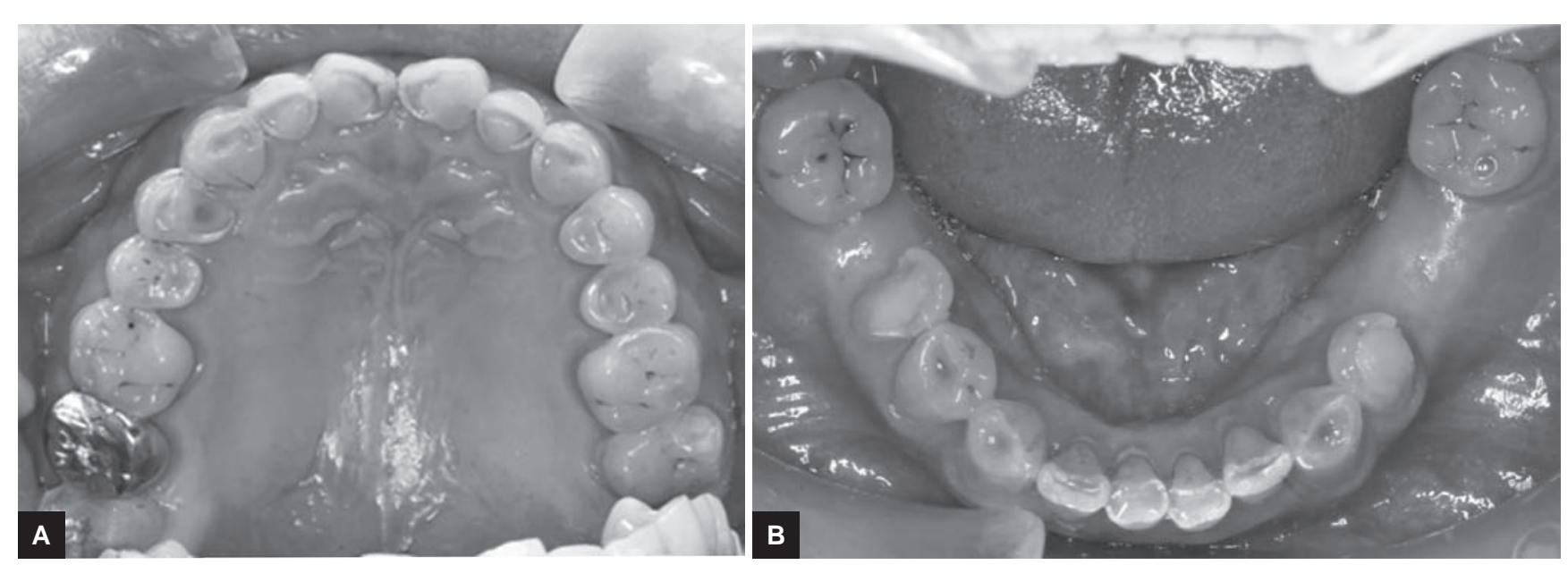

Figs 2A and B: (A) Postorthodontic upper occlusal view shows derotated 11, 21, and aligned teeth; and (B) postorthodontic lower occlusal views shows aligned lower anterior teeth

Treatment goals were to prevent further tooth surface loss, to improve alignment of teeth, to improve esthetics, and to improve oral function.

The patient first underwent a preventive phase, including dietary advice, oral hygiene instruction, scaling and prophylaxis, and topical fluoride treatment. A Michigan splint was delivered as a preventive measure to limit further tooth surface loss from bruxism. The patient was then referred to the Department of Orthodontics for consultation for realignment of teeth to facilitate future rehabilitation.

An orthodontic treatment using Invisalign (Align Technology, Inc., USA) was selected and accepted by both the clinicians and the patient. The study models were scanned to provide a precise three-dimensional (3D) computer image. From the image, a virtual treatment sequence of tooth movements was created using a software program called ClinCheck (Align Technology, Inc., USA), showing the pretreatment and posttreatment computer models. Both the treatment team and the patient agreed on the final position of teeth, a computer-aided manufacturing process fabricated a series of clear aligners. Each of these clear aligners was worn for approximately 2 weeks and 20 hours/day to move teeth in small

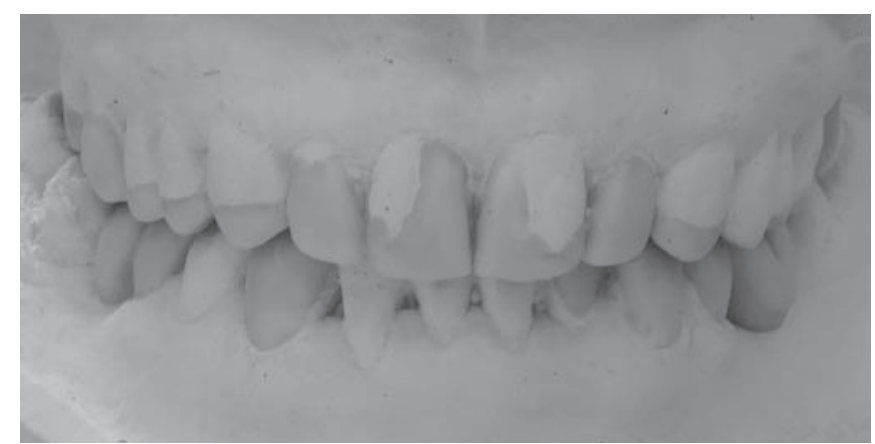

Fig. 3: Diagnostic wax-up increments until final results were achieved. A total of 26 sets of aligners were used. After 2 years of treatment, the alignment of teeth was completed (Figs 2A and B) and ready for restorative therapy.

Study models were recorded and mounted for analysis and the existing OVD was determined to increase by $3 \mathrm{~mm}$ to restore the esthetics and accommodate future dental restorations. A diagnostic wax-up was performed on duplicated study casts (Fig. 3), and an interim upper acrylic overlay denture was fabricated at the new OVD to facilitate transition to the new OVD (Fig. 4).

After 1 month, the dentition was prepared for fixed temporary restorations to assess the esthetics, speech, and comfort of the patient. All-ceramic crown and onlay preparations were made on the maxillary anterior teeth and premolars; full gold crown and onlay preparations were made on the maxillary molars. A silicone putty index of the diagnostic wax-up was used as a guide to determine the amount of tooth reduction required. At the planned increased OVD, the interocclusal space was sufficient for restorations without tooth preparation, therefore the palatal surfaces of upper anterior teeth and the occlusal surfaces of posterior teeth were just polished

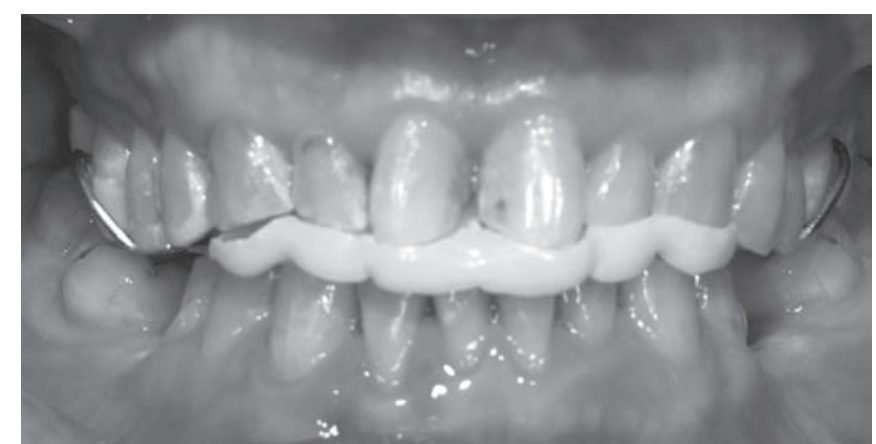

Fig. 4: Overlay denture delivered at increased OVD 
without significant tooth reduction to ensure no sharp edges or line angles are present. A chamfer preparation of $1 \mathrm{~mm}$ was prepared for all-ceramic restorations on the upper anterior and premolar teeth, with cast full-gold restorations on the upper molars. Autopolymerizing acrylic resin (ProTemp 4; 3M ESPE, Germany) was used chair-side with a vacuum-formed matrix to fabricate the restorations, they were spot-etched and cemented with resin cement, and the composite resin was placed on the lower arch as an interim restoration.

The patient was reviewed over 2 months, with some minor adjustments of the temporary restorations to optimize the occlusion and esthetics. Canine guidance and posterior disclusion on excursive mandibular movement was established for a "mutually protected occlusion." 15 The patient showed good adaptation to the new occlusion and was satisfied with the esthetics. A set of impressions with temporary restorations was taken. The occlusion of the temporary restorations was copied and transferred to a customized anterior guidance table using acrylic resin.

Fabrication of the definitive restoration was provided anteriorly, first with all-ceramic crowns (IPS e.max Press, Ivoclar Vivadent) on maxillary anterior teeth, which were cemented with resin luting cement (Variolink, Ivoclar Vivadent) and then direct composite (Esthet.X, Dentsply) built-up on the mandibular anterior teeth (Figs 5A and B) with the aid of a vacuum-formed matrix. The posterior teeth were then prepared on the right-hand side, followed by left-hand side. Adhesive gold onlays (tin-plated) were prepared on the upper molars and ceramic onlays (IPS e.max Press, Ivoclar Vivadent) on the upper second premolars. All-ceramic crowns were constructed for teeth 14 and 45, which had extensive coronal wear, and full gold crown was constructed for root-canal-treated 17. A resinbonded bridge was constructed to replace 46 , with 47 as an abutment and a fixed-movable conventional bridge was fabricated to replace 36 (Figs 6A to D).

As visible in the postoperative photographs, there was a mild relapse at the lower anterior segment, which was attributed to insufficient wearing of the removable retainers. Possible explanation may be that the patient could not wear the retainers for a few months after the teeth were temporized, as the retainers were not made to fit the provisional restorations. However, patient accepted the appearance and refused to start active orthodontic treatment again.

Clear overlay Essix retainers were fabricated for both arches after the definitive restorations to prevent further relapse and protect the restoration from nocturnal bruxism. The retainers were delivered for full-time wear during the first 6 months, and only at nighttime afterward. Regular maintenance was administered.

\section{DISCUSSION}

The management of advanced tooth wear is a restorative challenge for dental professionals. Identification and elimination of the causative factors of the tooth wear is of importance before active restorative treatment. Failure to eliminate the cause may lead to continued deterioration of teeth and definitive restorations.

The patient in this clinical report presented significant palatal tooth wear with large areas of dentin exposure with cupping on both posterior teeth and incisal edges and palatal surfaces of anterior teeth. The wear pattern together with the dietary habit of frequent consumption of sports drink correlated with the corrosive component in tooth wear. Sports drinks are reported to be acidic with typical $\mathrm{pH}$ value below $5.5{ }^{16}$ which is considered critical for loss of enamel. ${ }^{17}$ Furthermore, such drinks contains citric acid, which is considered one of the most erosive acids due to its chelating capacity, ${ }^{18}$ resulting in calcium sequestration from saliva and teeth. In addition, sugar in the sports drink can be metabolized by plaque microorganism forming organic acid, all of which contribute to the corrosion of dental hard tissues. ${ }^{19}$ Nocturnal bruxism was reported by the patient and, along with biocorrosion, was most likely the main etiological factor in this case. Dietary advice to reduce consumption of acidic foods and beverages and the

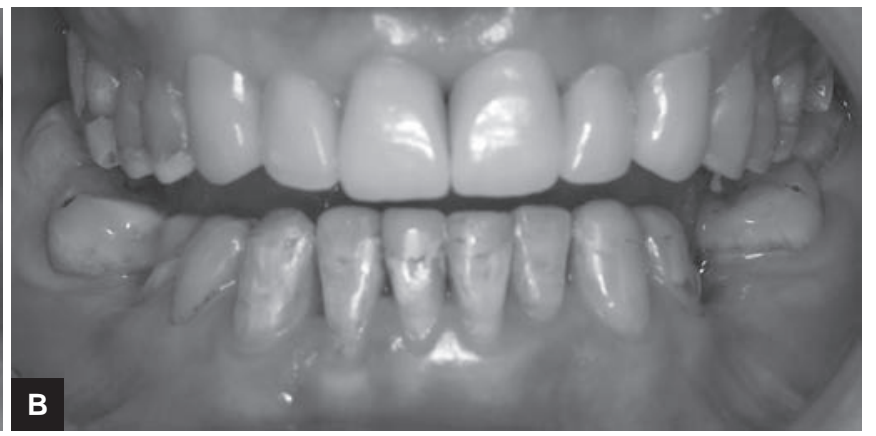



Figs 5A and B: (A) IPS e.max Press crowns cemented on maxillary anterior teeth; and (B) direct composite build-up on mandibular anterior teeth 

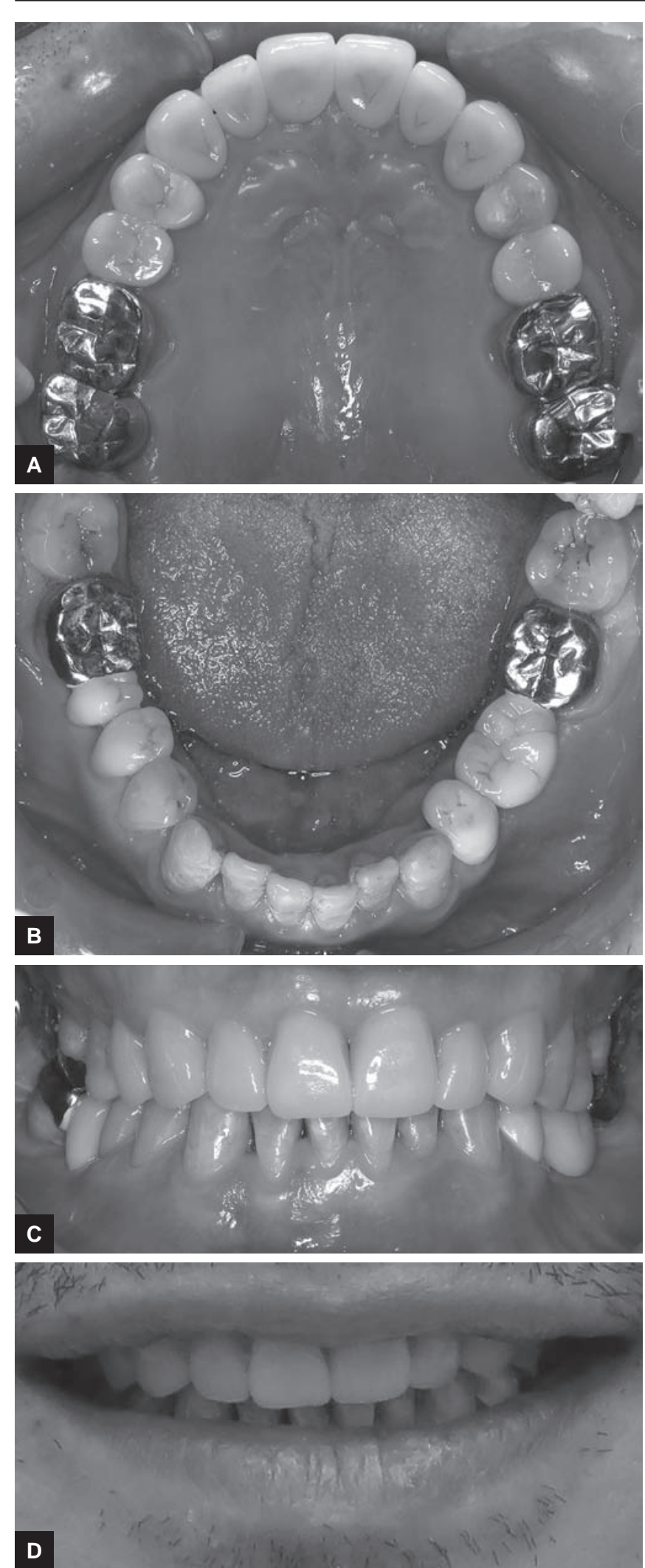

Figs 6A to D: (A) Postoperative upper occlusal view; (B) postoperative lower occlusal view; (C) postoperative frontal view; and (D) postoperative smile line

provision of Michigan splint to tooth surface loss were provided for long-term maintenance.

A reorganized occlusal scheme at an increased OVD was executed with posterior-anterior-posterior (PAP) approach. ${ }^{20}$ Posterior stability was first maintained by posterior provisional restorations, and the anterior guidance was then determined and definitive anterior crowns were constructed. After that, definitive posterior restorations were constructed on one side first, with the contralateral side maintaining the vertical dimension and the jaw position. Definitive posterior restorations were constructed after anterior restorations because the anterior guidance determined the posterior occlusal forms.

Direct composite resin restorations were used to restore lower anterior teeth with mild tooth wear that only involved incisal edges as a long-term restorative option. Composite resin restoration offers the advantages of conservative, acceptable aesthetic outcome, and easy to repair and adjust. Composite resin restoration had been used successfully on worn mandibular anterior teeth to increaseOVD. ${ }^{21}$ A promising 5-year survival rate of $78 \%$ was described for resin-bonded composite restorations placed in the anterior region for treatment of tooth wear. ${ }^{22}$ However, it is anticipated that long-term maintenance would be required. E.max (IPS e.max Press, Ivoclar Vivadent) was selected for restorations of upper anterior teeth and premolars, due to its superior esthetic and high strength. This has a flexural strength of $400 \mathrm{MPa} .{ }^{23} \mathrm{IPS}$ e.max consists of $70 \mathrm{vol} \%$ crystalline lithium disilicate fillers. Crowns made with lithium disilicate material have a reported survival rate of $94.8 \%$ after 8 years of service. ${ }^{24}$ Cast gold alloy was used for restorations of upper molars because they could be used in area with limited occlusal space, and they had a long history of satisfactory clinical performance. ${ }^{25}$

The patient requested fixed prosthodontic replacement of his lower missing teeth. The missing premolarsize 46 was replaced with a cantilever resin-bonded bridge, using 47 onlay as a retainer. Two-unit cantilevered resin-bonded bridges have been reported to achieve a $90 \%$ survival rate with a mean service life of 9.4 years, ${ }^{26}$ hence, good longevity could be expected. The missing 35 and 36 were replaced with a fixed-movable conventional bridge. A conventional bridge was chosen because the abutments needed full occlusal coverage; there was extensive tooth surface loss at 34 with insufficient coronal tooth structure existed for intracoronal restoration. Full occlusal coverage was required for 37 to harmonize the occlusal plane and to protect the tooth from further wear. Because of the different angulation of the abutments, a movable connector was placed on distal side of the mesial abutment. An option of an implant was excluded in this case because there was limited space at 46 , together with the patient's concern about the cost and surgical risk of implant treatment. Moreover, the patient was a smoker which is a risk factor for dental implant therapy. ${ }^{27}$ 
Interdisciplinary approach in the management of tooth wear was implemented in the current case. The patient in this case was first treated orthodontically using the Invisalign System before restoration. This approach not only reduced the amount of tooth reduction but also distributed space more evenly, thereby allowing space for better-proportioned restorations.

Invisalign is an orthodontic system to align teeth with a series of invisible and removable aligners. It was first developed to correct mild-to-moderate crowding, to close natural spaces, and to produce tipping. ${ }^{28}$ Invisalign is now used in more complex cases, such as those involving extraction and deep overbites. ${ }^{29,30}$ Advantages over conventional orthodontics is that it is free of unesthetic brackets and wires and thus more comfortable to the patient. Moreover, it is removable and facilitates oral hygiene procedures. Furthermore, the ClinCheck allows the clinician to design specific tooth movements and in this case, acts as a valuable communication tool between clinicians from the two disciplines.

A mild relapse of alignment of lower anterior teeth has been reported to occur, according to a systematic review, ${ }^{31}$ and satisfactory alignment was observed only in 30 to $50 \%$ of the patients in 10 years after the completion of orthodontic treatment, whereas this was further reduced to $10 \%$ after 20 years. Relapse can be managed by fixed or removable options. Fixed options include labial or lingual braces and archwires, and removable options include Hawley's retainer with active components to move the displaced teeth or invisible removable aligners, such as Invisalign. Since management of relapse is associated with additional costs and time, after considering the costbenefit ratio, the patient in this case accepted the small degree of relapse and preferred no active treatment for the relapsed lower anterior teeth.

Lifelong maintenance of the tooth wear is essential as all restorations will fail ultimately. It is important for the patient to fully understand the risk of restorative failure, and the maintenance needs from the outset, so as to manage expectations. ${ }^{25}$

\section{CONCLUSION}

Dentistry is varying with induction of modern science to practice dentistry. ${ }^{32}$

In this clinical report, generalized tooth wear with insufficient space for restoration, complicated by malalignment of teeth, was managed by way of in interdisciplinary approach, which allowed a conservative full-mouth rehabilitation. After orthodontic treatment, a reorganized restorative approach was undertaken at an increased vertical dimension providing an esthetic and functional solution. An occlusal night guard was provided to protect the dental restorations.

\section{CLINICAL SIGNIFICANCE}

Interdisciplinary approach allows a conservative management for patient with tooth wear complicated by malalignment of teeth.

\section{REFERENCES}

1. Smith BG, Knight JK. A comparison of patterns of tooth wear with aetiological factors. Br Dent J 1984 Jul 7;157(1):16-19.

2. Eccles JD. Erosion affecting the palatal surfaces of upper anterior teeth in young people. A report of 19 cases. Br Dent J 1982 Jun 1;152(11):375-378.

3. Grippo JO, Simring M, Coleman TA. Abfraction, abrasion, biocorrosion, and the enigma of noncarious cervical lesions: a 20-year perspective. J Esthet Restor Dent 2012 Feb;24(1): 10-25.

4. Schlueter N, Hardt M, Klimek J, Ganss C. Influence of the digestive enzymes trypsin and pepsin in vitro on the progression of erosion in dentin. Arch Oral Biol 2010 Apr;55(4): 294-299.

5. Hara AT, Ando M, Cury JA, Serra MC, González-Cabezas C, Zero DT. Influence of the organic matrix on root dentin erosion by citric acid. Caries Res 2005 Mar-Apr;39(2):134-138.

6. Kleter GA, Damen JJ, Everts V, Niehof J. The influence of the organic matrix on demineralization of bovine root dentin in vitro. J Dent Res 1994 Sep;73(9):1523-1529.

7. Grippo JO, Masi JV. The role of stress corrosion and piezoelectricity in the formation of root caries. In: Kenneth R, Foster PE, editors. Proceedings of the Thirteenth Annual Northeast Bioengineering Conference. Vol. 1. Philadelphia (PA): University of Pennsylvania; 1987.

8. Grippo JO, Masi JV. Role of biodental engineering factors (BEF) in the etiology of root caries. J Esthet Dent 1991 MarApr;3(2):71-76.

9. Kelleher M, Bishop K. Tooth surface loss: an overview. Br Dent J 1999 Jan 23;186(2):61-66.

10. Mehta SB, Banerji S, Millar BJ, Suarez-Feito JM. Current concepts on the management of tooth wear: part 2. Active restorative care 1: the management of localized tooth wear. Br Dent J 2012 Jan 27;212(2):73-82.

11. Dahl BL, Krogstad O, Karlsen K. An alternative treatment in cases with advanced localized attrition. J Oral Rehabil 1975 Jul;2(3):209-214.

12. Wassell RW, Steele JG. Considerations when planning occlusal rehabilitation: a review of the literature. Int Dent J 1998 Dec;48(6):571-581.

13. Carlsson GE, Ingervall BI, Kocak G. Effect of increasing vertical dimension on masticatory system in subjects with natural teeth. J Prosthet Dent 1979 Mar;41(3):284-289.

14. Norris RA, Brandt DJ, Crawford CH, Fallah M. Restorative and Invisalign ${ }^{\circledR}$ : a new approach. J Esthet Restor Dent 2002;14(4): 217-224.

15. Staurt CE, Stallard H. Concepts of occlusion. Dent Clin North Am 1963;7:591.

16. Pinto SC, Bandeca MC, Silva CN, Cavassim R, Borges AH, Sampaio JE. Erosive potential of energy drinks on the dentin surface. BMC Res Notes 2013 Feb 19;6:67. 
17. Zero DT. Etiology of dental erosion- extrinsic factors. Eur J Oral Sci 1996 Apr;104(2 Pt 2):162-177.

18. Burato EM, Andrade L, Rath IBS, Tames DR. Evaluation of the erosive potential of the Brazilian sport drinks to the dental hard tissues. Rev ABO Nac 2002;10:109-112.

19. Tahmassebi JF, Duggal MS, Malik-Kotru G, Curzon MEJ. Soft drinks and dental health: a review of the current literature. J Dent 2006 Jan;34(1):2-11.

20. Wise MD. Failure in the restored dentition: management and treatment. London: Quintessence; 1995. Chapter 10.

21. Poyser N, Porter R, Briggs P, Kelleher M. Demolition experts: management of the parafunctional patient: 2 Restorative management strategies. Dent Update 2007 Jun;34(5):262-268.

22. Smales R, Berekally T. Long-term survival of direct and indirect restorations placed for the treatment of advanced tooth wear. Eur J Prosthodont Restor Dent 2007 Mar;15(1):2-6.

23. Ma L, Guess PC, Zhang Y. Load-bearing properties of minimal-invasive monolithic lithium disilicate and zirconia occlusal onlays: finite element and theoretical analyses. Dent Mater 2013 Jul;29(7):742-751.

24. Gehrt M, Wolfart S, Rafai N, Reich S, Edelhoff D. Clinical results of lithium-disilicate crowns after up to 9 years of service. Clin Oral Investig 2013 Jan;17(1):275-284.

25. Mehta SB, Banerji S, Millar BJ, Suarez-Feito JM. Current concepts on the management of tooth wear: part 4 . An overview of the restorative techniques and dental materials commonly applied for the management of tooth wear. Br Dent J 2012 Feb 24;212(4):169-177.

26. Botelho MG, Ma X, Cheung GJ, Law RK, Tai MT, Lam WY. Long-term clinical evaluation of 211 two-unit cantilevered resinbonded fixed partial dentures. J Dent 2014 Jul;42(7):778-784.

27. Chrcanovic BR, Albrektsson T, Wennerberg A. Smoking and dental implants: a systematic review and meta-analysis. J Dent 2015 May;43(5):487-498.

28. Boyd RL, Miller RJ, Vlaskalic V. The Invisalign System in adult orthodontics for computer-assisted production of invisible appliances; mild crowding and space closure cases. J Clin Orthodont 2000;34(4):203-212.

29. Giancotti A, Garino F, Mampieri G. Lower incisor extraction treatment with the Invisalign ${ }^{\circledR}$ technique: three case reports. J Orthod 2015 Mar;42(1):33-44.

30. Giancotti A, Mampieri G, Greco M. Correction of deep bite in adults using the Invisalign system. J Clin Orthod 2008 Dec;42(12):719-726.

31. Yu Y, Sun J, Lai W, Wu T, Kishy S, Shi Z. Interventions for managing relapse of the lower front teeth after orthodontic treatment. Cochrane Database Syst Rev 2013 Sep 6;(9): CD008734

32. Saini R. Ozone therapy in dentistry: a strategic review. J Nat Sc Biol Med 2011; 2:151-153. 